\title{
Screening for Temperature Tolerant Genotypes and Genetic Parameter Studies in Chilli (Capsicum annuum L.)
}

\author{
Dileep Kumar*, G. D. Tembhurne, B. V. Vikas, V. Kulkarni, \\ R. V. Beldhadi, G. C. Shekar and B. Kisan \\ Department of Genetics and Plant Breeding, University of Agricultural Sciences, \\ Raichur-584102, Karnataka, India \\ *Corresponding author
}

\section{A B S T R A C T}

\section{Keywords \\ Chilli, Genetic parameters, Screening, Stress, Temperature tolerant}

Article Info

Accepted:

15 December 2019 Available Online: 20January 2020
Fifteen chilli genotypes were screened for temperature resilience during summer season in of 2017-18. The genotypes were subjected to two temperature levels, i.e. optimum temperature (inside the growth chamber) and summer temperature (in open field condition). The characters like plant height, fruit length, fruit weight, pedicel length, number of seeds per fruit, rate of photosynthesis, chlorophyll, pollen fertility, pollen viability and fruit set per cent were decreased and leaf proline, leaf phenol and ascorbic acid were increased under high temperature stress. However, variable response was observed for the traits oleoresin and capsaicin content depending upon the genotypes under temperature stress condition. Genotypes LCA-960, UARChH-42 and $9608 \mathrm{U}$ were found more temperature resilient in chilli. The genotype PBC-80 registered highly sensitive to temperature stress condition. Phenotypic coefficient of variation was more than genotypic coefficient of variation for all the characters studied. High heritability coupled with high genetic advance in per cent of mean was observed for characters like plant height, fruit length, fruit weight, pedicel length, number of seeds per fruit, rate of photosynthesis, chlorophyll, leaf proline, leaf phenol, ascorbic acid, oleoresin, capsaicin, pollen fertility, pollen viability and fruit set per cent.

\section{Introduction}

Chilli (Capsicum annuum L.) is an important commercial spice and vegetable crop. In chilli, flower abscission will be high if day temperature is in the range of $32-38^{\circ} \mathrm{C}$, whereas fruit retention will be maximal at 16$21^{\circ} \mathrm{C}$ day temperature (Demers et al., 1994).
Early summer to early rainy season is the lean period of production. Prevailing high temperature and shortage of soil moisture during early summer are the major factors limiting its cultivation during summer months. Area under cultivation for chilli during summer season was very much negligible in comparison with the cropping area during 
kharif season due to high temperature stress. Saha et al., (2010) reported that high temperature reduces percent fruit set, size of fruits as well as wide spectrum of both biochemical and physiological responses within the plant cells. Plant response to temperature stress is complex, involving adaptive changes and/ or deleterious effects. A plant strategy to cope with temperature stress normally involves a mixture of stress avoidance and tolerance 'strategies' that vary with genotype. In chilli the potential of genotypes to adapt under high temperature stress is not much known. Therefore, this study was undertaken identify the genotypes for high temperature stress, in chilli.

\section{Materials and Methods}

The present investigation was conducted at the Department of Genetics and Plant Breeding, College of Agriculture, Raichur during the year 2017-18. The experimental area was North Eastern dry region of Karnataka. The experimental materials for the present study comprises of 15 chilli genotypes viz., NM-1, Lipstick, Basavakalyan-1, PBC-80, ENT-1, G-4-S, Rajput, 9608U, JNB-1, LCA-960, UARChH-42, M-251, LCA-305, KA2-Long and BVC-37-1 with different genetic background. Considering the extremely hot summer the genotypes were subjected to screening during the month of March to May (Table 1). Seedlings were transplanted in PVC pipes for open field condition under high temperature and in pots using growth chamber under optimum temperature condition (Table 1). Completely Randomized Design (CRD) with three replications was used to conduct the experiment.

The data was recorded on five plants per genotype and averaged for statistical analysis for the traits plant height and photosynthesis rate during reproductive phase between 10:00 to 11 AM using Infrared Gas Analyzer, TPS-2 portable photosynthesis system version 2.01. However, characters like fruit length, fruit weight, pedicel length and number of seeds per fruit were taken from randomly selected five fruits per genotype in each replication. 2 per cent acetocarmine was used to study pollen fertility by counting properly stained pollen grains. The total number of pollen grains counted using three microscopic fields per slide under 10X magnification. The pollen viability study was conducted by hanging drop culture method and observations were recorded at three microscopic fields per slide after 24 hours of incubation by using compound microscope (10X). The pollen viability percentage was calculated as ratio of total viable pollen to the total number of pollens in three microscope fields and this procedure was repeated for 5 times to get accurate results. Per cent fruit set was recorded as the ratio of number of fruit set in each genotype to the total number of flowers tagged. Leaf proline content was determined by the method adopted by Bates et al., (1973). Leaf phenol content was determined by the method adopted by Bray and Thorpe (1954). Capsaicin content was estimated by the procedure proposed by Palacio (1977). Oleoresin content was using estimated using gravity method (Ranganna, 1977) and ascorbic acid determined by volumetric method. Chlorophyll content of leaf was measured at reproductive phase using SPAD during 10:00 to 11 AM.

Per cent change was calculated from control condition to stress condition by keeping control as cent per cent. The analysis of variance was calculated by using Federer (1977) method. The genotypic coefficient of variance (GCV) and phenotypic coefficient of variance (PCV) was estimated by the formulae given by Burton and De Vane (1953).

Heritability in broad sense was computed by the formulae given by Hanson et al., (1953) 
and the genetic advance over mean was determined as per the procedure of Johnson et al., (1955).

\section{Results and Discussion}

Reduction in mean performance was observed from control to stress condition for the characters like plant height, fruit length, fruit weight, pedicel length, number of seeds per fruit, rate of photosynthesis, chlorophyll content, pollen fertility, pollen viability and per cent fruit set (Table 2), similar findings were reported by Saha et al., (2010) for plant height and interpreted that under high temperature stress loss of cell turgor hampers cell elongation and plant growth which in turn reduces plant height. Dahal et al., (2006) found similar results for fruit length, fruit weight, pedicel length, number of seeds per fruit, rate of photosynthesis and chlorophyll content. Among the plants grown in open condition (under temperature stress) UARChH-42 produced maximum plant height, followed by Basavakalyan-1 which differed significantly, genotype LCA-960 showed maximum fruit length followed by UARChH-42 hybrid which were on par with each other, highest fruit weight was observed in LCA-960 followed by UARChH-42 and 9608U which differed significantly, maximum pedicel length was observed by genotype LCA-960 followed by $9608 \mathrm{U}$ and UARChH42 which differed significantly with each other, highest number of seeds per fruit were recorded in LCA-960, followed by UARChH42 and $9608 \mathrm{U}$ which were differed significantly with each other, genotype LCA960 showed higher photosynthetic rate, followed by hybrid UARChH-42 and 9608U which differed significantly with each other, genotype LCA-960 showed highest chlorophyll content followed by UARChH-42 and Rajput which differed significantly, pollen fertility was highest in LCA-960 followed by UARChH-42 and ENT-1, highest pollen viability was observed in LCA-960 followed by ENT-1 which differed significantly and highest fruit set was observed in LCA-960 followed by UARChH-42 and 9608U which differed significantly with each other. The traits like leaf proline content, leaf phenol content and ascorbic acid contents were increased under temperature stress condition (Table 2), similar results were reported by Navita et al., (2016). Genotype LCA-960 showed higher leaf proline content followed by $9608 \mathrm{U}$ and UARChH-42 which differed significantly with each other, highest leaf phenol content was exhibited by genotype LCA-960 followed by $9608 \mathrm{U}$ and UARChH42 which differed significantly with each other and highest ascorbic acid content was observed in LCA-960 followed by UARChH42 which was on par with each other. However, variable response was observed for the traits oleoresin and capsaicin content depending upon the genotypes under temperature stress condition (Table 2), similar results were reported by Alberto et al., (2013). Highest oleoresin content was observed in 9608U followed by NM-1 which was on par with each other, highest capsaicin content was observed by genotype Rajput followed by 9608U which was on par with the each other. Analysis of variance revealed that there was a significant difference among the genotypes for all the traits studied under both field and control conditions (Table 3). The magnitude of genetic variability was indicated by the genetic parameters viz., mean, range, phenotypic and genotypic coefficient of variability, heritability estimates and predicted genetic advance as per cent mean were computed for all the characters in two different growing conditions (Table 4). Estimates of PCV were higher than GCV for all the traits under both control as well as stress conditions indicating the environmental effect for the expression of these characters.

However, Under open field condition difference between phenotypic coefficient of variation $(\mathrm{PCV})$ and their corresponding 
genotypic coefficient of variation (GCV) were higher for the characters like per cent fruit set, average fruit weight, and for pollen viability indicating higher sensitivity to environmental effect. These findings are in close harmony with the result of Mohammed et al., (2001) for per cent fruit set and average fruit weight.

While, difference between the phenotypic coefficient of variation (PCV) and their corresponding genotypic coefficient of variation (GCV) were medium for the characters like plant height, fruit length, pedicel length, number of seeds per fruit, rate of photosynthesis, chlorophyll content, leaf proline content, leaf phenol content, oleoresin content and for pollen fertility indicating medium sensitivity to environment, these findings are similar with the result of Dipendera and Gautam (2002). However, difference between phenotypic coefficient of variation (PCV) and their corresponding genotypic coefficient of variation (GCV) were much narrow for the characters like ascorbic content and capsaicin content indicating low sensitivity to environment, consequently higher role of genetic factors influencing the character expression.

The characters with almost equal values of GCV and PCV can be considered as stable.
Genotypes grown under controlled condition in growth chamber showed much narrow difference between phenotypic coefficient of variation $(\mathrm{PCV})$ and their corresponding genotypic coefficient of variation (GCV) for all the characters indicating low environmental influence, consequently higher role of genetic factors influencing the character expression. Heritability and genetic advance as per cent mean were higher for all the characters under control as well as high temperature stress conditions, indicating all the characters studied may be improved through selection to identify the genotypes suitable for high temperature stress. Characters like plant height, fruit length, fruit weight, number of seeds per fruit, pedicel length, rate of photosynthesis, chlorophyll content, leaf proline content, leaf phenol content, oleoresin content, capsaicin content, ascorbic acid content, pollen fertility, pollen viability and per cent fruit set showed high heritability and high genetic advance as per cent mean. High heritability for these characters indicates high response to selection and high genetic advance values have explained the predominance of additive gene action and selection for these traits can be carried out in early generation.

Table.1 Climatic conditions observed during reproductive stage of chilli grown in open field condition in the year 2018

\begin{tabular}{|c|c|c|c|c|c|c|c|c|}
\hline \multirow[t]{2}{*}{ Sl.No. } & \multicolumn{8}{|c|}{ Open field condition ( $\mathrm{PVC}$ pipes) } \\
\hline & Month & $\underset{\left({ }^{\circ} \mathrm{C}\right)}{T \text { max }}$ & $\begin{array}{c}T_{\text {min }} \text { min } \\
\left({ }^{\circ} \mathrm{C}\right)\end{array}$ & $\begin{array}{c}\text { Mean } \\
\left({ }^{\circ} \mathrm{C}\right)\end{array}$ & $\begin{array}{c}\text { RH I } \\
(\%)\end{array}$ & $\begin{array}{c}\text { RH II } \\
(\%)\end{array}$ & $\begin{array}{c}\text { Mean RH } \\
(\%)\end{array}$ & $\begin{array}{c}\text { SS } \\
(\text { hrs })\end{array}$ \\
\hline 1 & March & 36.8 & 22.4 & 29.6 & 55 & 29 & 42 & 6.6 \\
\hline 2 & April & 38.9 & 26.2 & 32.5 & 57 & 28 & 42 & 7.2 \\
\hline 3 & May & 39.7 & 27.6 & 33.7 & 62 & 32 & 47 & 6.2 \\
\hline \multicolumn{9}{|c|}{ Growth chamber (control condition) } \\
\hline 1 & March & 29 & 21 & 25 & 70 & 65 & 67.5 & 8 \\
\hline 2 & April & 29 & 21 & 25 & 70 & 65 & 67.5 & 8 \\
\hline 3 & May & 28 & 20 & 24 & 70 & 65 & 67.5 & 9 \\
\hline
\end{tabular}


Table.2 Effect of temperature stress on different traits of chilli under temperature stress condition

\begin{tabular}{|c|c|c|c|c|c|c|c|c|c|c|c|c|c|c|c|c|c|c|c|c|c|c|}
\hline \multirow[t]{2}{*}{$\begin{array}{l}\text { SI. } \\
\text { No. }\end{array}$} & \multirow[t]{2}{*}{ Genotypes } & \multicolumn{3}{|c|}{ Plant height (cm) } & \multicolumn{3}{|c|}{ Fruit length (cm) } & \multicolumn{3}{|c|}{ Fruit weight (g) } & \multicolumn{3}{|c|}{ Pedicel length $(\mathrm{cm})$} & \multicolumn{3}{|c|}{ Number of seeds/ fruit } & \multicolumn{3}{|c|}{$\begin{array}{l}\text { Rate of photosynthesis } \\
(\mu \mathrm{mol} \text { CO2 m-2 s-1) }\end{array}$} & \multicolumn{3}{|c|}{$\begin{array}{c}\text { Leaf proline content } \\
(\mathrm{mg} / \mathrm{g} \mathrm{FW})\end{array}$} \\
\hline & & $\mathrm{CC}$ & TS & PD/PI & $\mathrm{CC}$ & TS & PD/PI & $\mathrm{CC}$ & TS & PD/PI & $\mathrm{CC}$ & TS & PD/PI & $\mathrm{CC}$ & TS & PD/PI & $\mathrm{CC}$ & TS & $\mathbf{P D} / \mathbf{P I}$ & $\mathrm{CC}$ & TS & PD/PI \\
\hline 1 & NM-1 & 64.15 & 56.83 & -11.41 & 6.18 & 5.55 & -10.19 & 0.89 & 0.66 & -25.84 & 2.53 & 2.10 & -17.00 & 34.07 & 29.27 & -14.09 & 22.27 & 15.30 & -31.29 & 0.74 & 0.99 & 33.96 \\
\hline 2 & Lipstick & 66.86 & 53.03 & -20.69 & 3.93 & 3.35 & -14.76 & 0.80 & 0.63 & -21.25 & 2.62 & 2.20 & -16.03 & 37.33 & 34.47 & -7.66 & 18.61 & 11.76 & -36.81 & 0.90 & 2.03 & 124.39 \\
\hline 3 & Basavakalyan-1 & 67.46 & 60.50 & -10.32 & 6.95 & 6.28 & -9.64 & 1.09 & 0.85 & -22.02 & 1.65 & 1.30 & -21.21 & 49.36 & 40.34 & -18.27 & 18.58 & 11.44 & -38.42 & 0.64 & 1.27 & 99.07 \\
\hline 4 & PBC-80 & 56.21 & 25.83 & -54.05 & 3.33 & 0.00 & 0.00 & 0.55 & 0.00 & 0.00 & 1.35 & 00.00 & 0.00 & 20.40 & 0.00 & 0.00 & 17.90 & 10.61 & -40.72 & 0.56 & 0.64 & 14.92 \\
\hline 5 & ENT-1 & 61.86 & 52.33 & -15.41 & 4.19 & 3.57 & -14.80 & 0.84 & 0.66 & -21.43 & 2.72 & 2.29 & -15.81 & 39.67 & 32.95 & -16.94 & 18.81 & 13.28 & -29.40 & 0.70 & 0.94 & 32.77 \\
\hline 6 & G-4-S & 59.93 & 50.61 & -15.55 & 4.69 & 4.25 & -9.38 & 0.52 & 0.36 & -30.77 & 2.01 & 1.60 & -20.40 & 31.35 & 26.73 & -14.74 & 23.69 & 17.23 & -27.27 & 0.86 & 1.37 & 58.57 \\
\hline 7 & Rajput & 64.60 & 53.21 & -17.63 & 3.97 & 3.37 & -15.11 & 0.83 & 0.65 & -21.68 & 1.91 & 1.50 & -21.47 & 39.03 & 31.20 & -20.06 & 22.47 & 16.82 & -25.14 & 0.79 & 2.82 & 255.22 \\
\hline 8 & $9608 \mathrm{U}$ & 59.89 & 54.31 & -9.32 & 7.07 & 6.53 & -7.64 & 0.98 & 0.86 & -12.24 & 3.58 & 3.19 & -10.89 & 44.53 & 41.43 & -6.96 & 24.72 & 18.44 & -25.40 & 1.06 & 4.08 & 284.46 \\
\hline 9 & JNB-1 & 63.28 & 50.36 & -20.42 & 3.92 & 3.61 & -7.91 & 0.86 & 0.65 & -24.42 & 3.01 & 2.60 & -13.62 & 39.53 & 35.88 & -9.23 & 22.06 & 15.91 & -27.88 & 1.02 & 1.27 & 24.51 \\
\hline 10 & LCA-960 & 63.06 & 57.31 & -9.12 & 7.40 & 6.98 & -5.68 & 1.08 & 0.99 & -8.33 & 3.74 & 3.39 & -9.36 & 61.50 & 57.13 & -7.11 & 24.72 & 19.67 & -20.42 & 1.08 & 4.30 & 298.51 \\
\hline 11 & UARChH-42 & 74.65 & 66.98 & -10.27 & 7.08 & 6.71 & -5.23 & 1.21 & 0.89 & -26.45 & 3.34 & 2.99 & -10.48 & 67.57 & 54.20 & -19.79 & 25.25 & 19.56 & -22.53 & 1.00 & 4.00 & 296.82 \\
\hline 12 & M-251 & 66.64 & 57.62 & -13.54 & 5.84 & 5.31 & -9.08 & 0.83 & 0.60 & -27.71 & 1.55 & 1.20 & -22.58 & 42.47 & 38.73 & -8.81 & 18.91 & 12.87 & -31.94 & 1.02 & 3.61 & 254.16 \\
\hline 13 & LCA-305 & 57.37 & 47.71 & -16.84 & 6.07 & 5.50 & -9.39 & 1.03 & 0.79 & -23.30 & 2.09 & 1.70 & -18.66 & 46.20 & 34.28 & -25.80 & 24.71 & 15.91 & -35.61 & 0.81 & 3.23 & 294.74 \\
\hline 14 & KA2-Long & 59.37 & 51.31 & -13.58 & 5.87 & 5.17 & -11.62 & 1.02 & 0.82 & -19.61 & 2.41 & 2.00 & -17.01 & 43.20 & 40.29 & -6.74 & 19.22 & 14.49 & -24.60 & 0.85 & 2.76 & 222.55 \\
\hline \multirow[t]{3}{*}{15} & BVC-37-1 & 61.50 & 48.55 & -21.06 & 4.93 & 4.36 & -11.56 & 0.71 & 0.55 & -22.54 & 1.67 & 1.32 & -20.96 & 31.27 & 27.66 & -11.54 & 20.64 & 15.30 & -25.87 & 0.91 & 1.00 & 10.03 \\
\hline & C.D 1\% & 4.59 & 3.69 & & 0.19 & 0.11 & & 0.08 & 0.06 & & 0.19 & 0.11 & & 2.27 & 2.14 & & 1.13 & 1.00 & & 0.04 & 0.14 & \\
\hline & C.V. & 3.13 & 2.24 & & 2.59 & 3.54 & & 3.92 & 4.20 & & 2.58 & 3.59 & & 2.42 & 2.27 & & 2.36 & 2.95 & & 1.87 & 2.10 & \\
\hline
\end{tabular}

\section{CC- Control condition; TS- Temperature stress; PD/PI - Per cent decrease or per cent increase}

\begin{tabular}{|c|c|c|c|c|c|c|c|c|c|c|c|c|c|c|c|c|c|c|c|c|c|c|c|c|c|}
\hline \multirow{3}{*}{$\begin{array}{l}\text { Sl. } \\
\text { No. }\end{array}$} & \multirow{3}{*}{ Genotypes } & \multirow{2}{*}{\multicolumn{3}{|c|}{$\begin{array}{l}\text { Leaf phenol content } \\
(\mathrm{mg} / \mathrm{g} \text { FW) }\end{array}$}} & \multirow{2}{*}{\multicolumn{3}{|c|}{$\begin{array}{c}\text { Chlorophyll content } \\
(\mathbf{m g} / \mathbf{g})\end{array}$}} & \multirow{2}{*}{\multicolumn{3}{|c|}{ Oleoresin $(\%)$}} & \multirow{2}{*}{\multicolumn{3}{|c|}{ Ascorbic acid (mg/g) }} & \multirow{2}{*}{\multicolumn{3}{|c|}{$\begin{array}{c}\text { Capsaicin content } \\
(\%)\end{array}$}} & \multirow{2}{*}{\multicolumn{3}{|c|}{ Pollen fertility (\%) }} & & & Table & (Contd. & & \\
\hline & & & & & & & & & & & & & & & & & & & & \multicolumn{3}{|c|}{ Pollen viability $(\%)$} & \multicolumn{3}{|c|}{ Per cent fruit set (\%) } \\
\hline & & $\mathrm{CC}$ & TS & PD/PI & $\mathbf{C C}$ & TS & PD/PI & $\mathrm{CC}$ & TS & PD/PI & $\mathrm{CC}$ & TS & PD/PI & $\mathrm{CC}$ & TS & PD/PI & $\mathrm{CC}$ & TS & PD/PI & $\mathrm{CC}$ & TS & PD/PI & CC & TS & PD/PI \\
\hline 1 & NM-1 & 13.09 & 18.18 & 38.88 & 0.81 & 0.77 & -4.93 & 10.11 & 11.70 & 25.61 & 25.61 & 31.57 & 23.27 & 0.23 & 0.22 & -4.68 & 61.91 & 53.79 & -13.12 & 37.54 & 25.70 & -31.54 & 63.33 & 24.33 & -61.58 \\
\hline 2 & Lipstick & 13.85 & 17.71 & 27.87 & 0.88 & 0.83 & -5.53 & 10.20 & 12.33 & 20.88 & 30.41 & 32.68 & 7.46 & 0.29 & 0.28 & -3.74 & 62.45 & 55.28 & -11.48 & 34.11 & 27.57 & -19.17 & 64.00 & 25.00 & -60.94 \\
\hline 3 & $\begin{array}{c}\text { Basavakalyan- } \\
1 \\
\end{array}$ & 14.62 & 17.39 & 18.95 & 0.75 & 0.73 & -3.35 & 10.40 & 12.10 & 16.40 & 30.41 & 44.92 & 47.71 & 0.32 & 0.29 & -7.45 & 66.20 & 54.67 & -17.42 & 34.80 & 27.03 & -22.33 & 66.00 & 17.67 & -73.23 \\
\hline 4 & PBC-80 & 11.95 & 17.26 & 44.44 & 0.63 & 0.48 & -23.12 & 12.18 & 00.00 & 00.00 & 34.16 & 00.00 & 00.00 & 0.34 & 0.00 & 0.00 & 56.53 & 00.00 & 0.00 & 30.76 & 00.00 & 0.00 & 57.67 & 00.00 & 0.00 \\
\hline 5 & ENT-1 & 12.51 & 16.49 & 31.81 & 0.69 & 0.55 & -20.82 & 10.05 & 10.31 & 2.58 & 26.51 & 33.62 & 26.82 & 0.25 & 0.24 & -4.76 & 66.64 & 58.51 & -12.20 & 55.33 & 31.61 & -42.87 & 62.33 & 28.00 & -55.08 \\
\hline 6 & G-4-S & 12.52 & 13.13 & 4.87 & 0.96 & 0.93 & -3.16 & 12.32 & 11.44 & -7.14 & 34.02 & 40.11 & 17.90 & 0.30 & 0.32 & 5.84 & 64.99 & 51.36 & -20.97 & 35.87 & 28.90 & -19.43 & 64.33 & 21.00 & -67.36 \\
\hline 7 & Rajput & 12.35 & 17.61 & 42.59 & 0.94 & 0.91 & -3.35 & 10.38 & 12.73 & 22.6 & 27.51 & 36.91 & 34.17 & 0.23 & 0.33 & 22.43 & 66.25 & 51.27 & -22.61 & 31.96 & 25.70 & -19.59 & 65.33 & 20.00 & -69.39 \\
\hline 8 & $9608 \mathrm{U}$ & 17.39 & 19.14 & 10.06 & 0.92 & 0.88 & -4.15 & 12.68 & 12.71 & 0.23 & 33.61 & 43.52 & 29.49 & 0.36 & 0.33 & -6.11 & 65.52 & 56.44 & -13.86 & 31.45 & 29.33 & -6.74 & 75.00 & 33.33 & -55.56 \\
\hline 9 & JNB-1 & 11.85 & 14.86 & 25.40 & 0.74 & 0.68 & -8.40 & 10.47 & 11.57 & 10.55 & 26.17 & 37.06 & 41.61 & 0.24 & 0.26 & 5.24 & 66.29 & 57.44 & -13.35 & 36.07 & 29.85 & -17.24 & 62.33 & 26.33 & -57.76 \\
\hline 10 & LCA-960 & 16.91 & 19.51 & 15.37 & 1.08 & 1.04 & -4.25 & 12.05 & 11.74 & -2.57 & 34.26 & 46.42 & 35.49 & 0.27 & 0.28 & 5.49 & 72.21 & 66.42 & -8.02 & 44.39 & 35.08 & -20.97 & 75.33 & 42.00 & -44.25 \\
\hline 11 & UARChH-42 & 15.35 & 18.19 & 18.50 & 1.03 & 0.98 & -4.18 & 11.82 & 12.66 & 7.15 & 27.11 & 45.42 & 67.54 & 0.22 & 0.21 & -5.26 & 82.96 & 59.24 & -28.59 & 58.45 & 31.36 & -46.35 & 84.33 & 37.00 & -56.12 \\
\hline 12 & $\mathrm{M}-251$ & 11.64 & 12.13 & 4.20 & 0.92 & 0.85 & -8.32 & 11.27 & 12.30 & 9.14 & 30.31 & 30.56 & 0.82 & 0.24 & 0.23 & -2.45 & 67.71 & 46.92 & -30.70 & 30.49 & 23.13 & -24.14 & 62.67 & 14.33 & -77.13 \\
\hline 13 & LCA-305 & 12.64 & 13.12 & 3.80 & 0.94 & 0.91 & -3.34 & 12.03 & 11.30 & -6.07 & 33.11 & 36.21 & 9.36 & 0.21 & 0.22 & 5.58 & 62.32 & 56.11 & -9.96 & 36.23 & 27.76 & -23.38 & 66.00 & 22.00 & -66.67 \\
\hline 14 & KA2-Long & 11.70 & 14.94 & 27.69 & 0.80 & 0.77 & -4.84 & 10.78 & 12.39 & 14.93 & 25.30 & 42.52 & 68.06 & 0.34 & 0.32 & -5.29 & 62.22 & 52.99 & -14.83 & 35.82 & 25.88 & -27.75 & 68.67 & 24.00 & -65.05 \\
\hline \multirow[t]{3}{*}{15} & BVC-37-1 & 12.84 & 15.10 & 17.60 & 0.97 & 0.95 & -2.61 & 10.16 & 10.45 & 2.85 & 25.27 & 36.46 & 44.28 & 0.33 & 0.22 & -32.33 & 67.35 & 53.54 & -20.50 & 33.84 & 25.15 & -25.68 & 61.00 & 17.00 & -72.13 \\
\hline & C.D 1\% & 0.60 & 0.66 & & 0.04 & 0.03 & & 0.34 & 0.48 & & 0.89 & 1.87 & & 0.02 & 0.02 & & 4.67 & 4.49 & & 3.61 & 2.50 & & 5.13 & 2.14 & \\
\hline & C.V. & 1.83 & 1.90 & & 1.40 & 1.70 & & 1.80 & 1.71 & & 1.10 & 1.77 & & 2.01 & 3.22 & & 3.14 & 3.90 & & 4.23 & 4.25 & & 3.43 & 4.07 & \\
\hline
\end{tabular}

\section{CC- Control condition}

TS- Temperature stress

PD/PI - Per cent decrease or per cent increase 
Table.3 Analysis of variance for quantitative and qualitative traits in chilli

\begin{tabular}{|c|c|c|c|c|c|c|c|c|c|c|c|c|c|c|c|c|}
\hline $\begin{array}{l}\text { Source of } \\
\text { Variation }\end{array}$ & df & $\mathbf{X}_{1}$ & $\mathbf{X}_{2}$ & $\mathbf{X}_{3}$ & $\mathbf{X}_{4}$ & $\mathbf{X}_{5}$ & $X_{6}$ & $\mathbf{X}_{7}$ & $\mathbf{X}_{8}$ & $X_{9}$ & $\mathbf{X}_{10}$ & $X_{11}$ & $X_{12}$ & $\mathbf{X}_{13}$ & $X_{14}$ & $\mathbf{X}_{15}$ \\
\hline $\begin{array}{l}\text { Treatments } \\
\text { (control) }\end{array}$ & 14 & $64.55 * *$ & $5.644 * *$ & $0.124 * *$ & $1.72 * *$ & $410.9 * *$ & $48.06^{* *}$ & $0.049 * *$ & $20.16^{* *}$ & $0.050 * *$ & $4.528 * *$ & $46.28 * *$ & $0.005 * *$ & $102.7 * *$ & $215.5^{* *}$ & $\begin{array}{c}140.03 * \\
*\end{array}$ \\
\hline $\begin{array}{l}\text { Treatments } \\
\text { (stress) }\end{array}$ & 14 & $\begin{array}{c}236.79 * \\
*\end{array}$ & $9.750 * *$ & $0.177 * *$ & $2.342 * *$ & $\begin{array}{l}508.01 * \\
*\end{array}$ & $32.90 * *$ & $6.742 * *$ & $19.40 * *$ & $0.074 * *$ & $19.15^{* *}$ & $250.9 * *$ & $0.014 * *$ & $668.9 * *$ & $185.9 * *$ & $296.9 * *$ \\
\hline $\begin{array}{c}\text { Error } \\
\text { (control) }\end{array}$ & 30 & 4.188 & 0.020 & 0.001 & 0.008 & 1.022 & 0.252 & 0.0001 & 0.0001 & 0.042 & 0.038 & 0.092 & 0.0001 & 4.328 & 2.584 & 5.222 \\
\hline $\begin{array}{c}\text { Error } \\
\text { (stress) }\end{array}$ & 30 & 2.703 & 0.028 & 0.001 & 0.003 & 0.906 & 0.198 & 0.0001 & 0.002 & 0.050 & 0.033 & 0.404 & 0.000 & 3.997 & 26.31 & 0.911 \\
\hline $\begin{array}{l}\mathrm{X}_{1}: \mathrm{P} \\
\mathrm{X}_{2}: \mathrm{F} \\
\mathrm{X}_{3}: \mathrm{F} \\
\mathrm{X}_{4}: \mathrm{P} \\
\mathrm{X}_{5}: \mathrm{P}\end{array}$ & $\begin{array}{l}\text { nt he } \\
\text { it le } \\
\text { it we } \\
\text { licel } \\
\text { mbe }\end{array}$ & $\begin{array}{l}\text { ght }(\mathrm{cm}) \\
\text { th }(\mathrm{cm}) \\
\text { ght }(\mathrm{g}) \\
\text { ength }(\mathrm{cm}) \\
\text { of seeds/fr }\end{array}$ & & & & $\begin{array}{l}\mathrm{X}_{6}: \text { Rate of } \\
\mathrm{X}_{7}: \text { Leaf } \mathrm{p} \\
\mathrm{X}_{8}: \text { Leaf } \mathrm{pl} \\
\mathrm{X}_{9}: \text { Chloro } \\
\mathrm{X}_{10}: \text { Oleor }\end{array}$ & $\begin{array}{l}\text { photosyn } \\
\text { line cont } \\
\text { enol cont } \\
\text { hyll cont } \\
\text { in conter }\end{array}$ & $\begin{array}{l}\text { esis }(\mu \mathrm{mo} \\
\text { ht }(\mathrm{mg} / \mathrm{g}) \\
\text { tt }(\mathrm{mg} / \mathrm{g}) \\
\text { ht }(\mathrm{mg} / \mathrm{g}) \\
(\%)\end{array}$ & $\mathrm{nol}^{-1} \mathrm{C}$ & $\mathrm{n} / \mathrm{s})$ & & $\begin{array}{l}\text { A } \\
X \\
X \\
X \\
X\end{array}$ & $\begin{array}{l}\text { Ascorbi } \\
\text { : Capsaic } \\
\text { : Pollen f } \\
\text { : Pollen } \\
\text { : Per cent }\end{array}$ & $\begin{array}{l}\text { acid cont } \\
\text { l content } \\
\text { tility }(\%) \\
\text { ability (\% } \\
\text { ruit set. }\end{array}$ & $\begin{array}{l}\mathrm{nt}(\mathrm{mg} / \mathrm{g}) \\
\%)\end{array}$ & \\
\hline
\end{tabular}

Table.4 Genetic parameters for quantitative and qualitative characters in chilli

\begin{tabular}{|c|c|c|c|c|c|c|c|c|c|c|c|c|c|c|c|c|}
\hline \multirow{2}{*}{ Particulars } & \multicolumn{2}{|c|}{ MEAN } & \multicolumn{2}{|c|}{ MIN } & \multicolumn{2}{|c|}{ MAX } & \multicolumn{2}{|c|}{ GCV (\%) } & \multicolumn{2}{|c|}{ PCV (\%) } & \multicolumn{2}{|c|}{$\mathbf{h}^{2}(\%)$} & \multicolumn{2}{|c|}{ GA $(5 \%)$} & \multicolumn{2}{|c|}{ GAM (5\%) } \\
\hline & Control & Stress & Control & Stress & Control & Stress & Control & Stress & Control & Stress & Control & Stress & Control & Stress & Control & Stress \\
\hline $\mathrm{X1}$ & 63.1 & 52.4 & 56.2 & 25.8 & 74.5 & 67.0 & 7.2 & 16.5 & 7.6 & 17.8 & 88.4 & 85.8 & 8.8 & 16.5 & 13.9 & 31.5 \\
\hline $\mathrm{X} 2$ & 6.3 & 05.8 & 3.3 & 3.4 & 7.4 & 7.0 & 25.1 & 37.6 & 25.7 & 39.7 & 95.4 & 89.9 & 2.7 & 3.5 & 50.5 & 73.5 \\
\hline $\mathbf{X 3}$ & 0.8 & 0.7 & 0.5 & 0.4 & 1.2 & 1.0 & 23.5 & 35.3 & 25.7 & 39.9 & 83.5 & 78.2 & 0.4 & 0.5 & 44.2 & 64.2 \\
\hline $\mathrm{X} 4$ & 02.4 & 01.9 & 1.4 & 1.2 & 3.7 & 3.4 & 31.2 & 44.6 & 31.9 & 46.2 & 95.7 & 93.3 & 1.5 & 1.7 & 62.9 & 88.7 \\
\hline X5 & 41.8 & 34.9 & 20.4 & 26.7 & 67.6 & 57.1 & 27.7 & 36.8 & 28.4 & 38.0 & 95.6 & 93.5 & 23.4 & 25.6 & 55.9 & 73.3 \\
\hline X6 & 21.2 & 15.1 & 17.9 & 10.6 & 25.3 & 19.7 & 18.8 & 21.5 & 19.0 & 22.9 & 98.6 & 88.4 & 8.2 & 6.3 & 38.5 & 41.7 \\
\hline X7 & 0.8 & 02.7 & 0.6 & 0.7 & 1.1 & 5.3 & 18.0 & 66.8 & 18.1 & 67.5 & 98.9 & 98.1 & 0.3 & 3.7 & 37.0 & 136.3 \\
\hline X8 & 12.2 & 16.2 & 11.6 & 12.1 & 17.4 & 19.5 & 25.7 & 19.2 & 25.7 & 19.3 & 98.6 & 98.5 & 6.5 & 6.4 & 52.8 & 39.5 \\
\hline X9 & 00.9 & 00.8 & 0.6 & 0.5 & 1.1 & 1.0 & 14.8 & 18.6 & 14.9 & 20.5 & 97.9 & 82.0 & 0.3 & 0.3 & 30.1 & 34.6 \\
\hline X10 & 09.9 & 11.3 & 10.0 & 10.3 & 12.7 & 12.7 & 13.8 & 29.8 & 13.9 & 30.5 & 98.3 & 95.3 & 3.0 & 6.2 & 28.0 & 59.8 \\
\hline X11 & 28.8 & 35.9 & 25.3 & 30.6 & 34.3 & 46.4 & 16.7 & 31.1 & 16.7 & 31.4 & 98.6 & 98.3 & 9.9 & 22.8 & 34.3 & 63.5 \\
\hline $\mathrm{X12}$ & 00.3 & 00.3 & 0.21 & 0.21 & 0.4 & 0.33 & 17.8 & 32.4 & 17.9 & 32.8 & 98.7 & 97.6 & 0.1 & 0.2 & 36.4 & 65.9 \\
\hline X13 & 66.1 & 51.6 & 56.5 & 46.9 & 82.9 & 66.4 & 8.8 & 28.5 & 9.0 & 29.8 & 94.3 & 91.9 & 11.6 & 29.0 & 17.5 & 56.3 \\
\hline X14 & 37.8 & 26.3 & 30.5 & 23.1 & 58.5 & 35.1 & 22.3 & 29.1 & 22.7 & 31.5 & 96.5 & 85.4 & 17.0 & 14.6 & 45.1 & 55.5 \\
\hline X15 & 66.6 & 23.5 & 57.7 & 14.3 & 84.3 & 42.0 & 10.1 & 41.2 & 10.7 & 45.0 & 89.1 & 84.0 & 13.0 & 18.2 & 19.6 & 77.8 \\
\hline \multicolumn{17}{|c|}{$\mathrm{X}_{1}$ : Plant height $(\mathrm{cm})$} \\
\hline \multicolumn{9}{|c|}{$\mathrm{X}_{4}$ : Pedicel length $(\mathrm{cm})$} & \multicolumn{4}{|c|}{$\mathrm{X}_{6}:$ Rate of photosynthesis } & & & & \\
\hline \multicolumn{5}{|c|}{$\mathrm{X}_{7}$ : Leaf proline content $(\mathrm{mg} / \mathrm{g})$} & \multicolumn{4}{|c|}{$\mathrm{X}_{8}$ : Leaf phenol content $(\mathrm{mg} / \mathrm{g})$} & \multicolumn{4}{|c|}{$\mathrm{X}_{9}$ : Chlorophyll content $(\mathrm{mg} / \mathrm{g})$} & & & & \\
\hline \multicolumn{5}{|c|}{$\mathrm{X}_{10}:$ Oleoresin content $(\%)$} & \multicolumn{4}{|c|}{$\mathrm{X}_{11}:$ Ascorbic acid content $(\mathrm{mg} / \mathrm{g})$} & \multicolumn{4}{|c|}{$\mathrm{X}_{12}:$ Capsaicin content $(\%)$} & & & & \\
\hline \multicolumn{5}{|c|}{$\mathrm{X}_{13}:$ Pollen fertility $(\%)$} & \multicolumn{4}{|c|}{$\mathrm{X}_{14}:$ Pollen viability $(\%)$} & \multicolumn{4}{|c|}{$\mathrm{X}_{15}$ : Per cent fruit set } & & & & \\
\hline
\end{tabular}


Heat tolerance is a multigenic character, numerous biochemical and metabolic traits are also involved in the development and maintenance of thermo tolerance. As photosynthesis and reproductive development are the most sensitive physiological processes to temperature stress in chilli, the temperature tolerant variety will be usually characterized by higher photosynthetic rates reflected in stay-green leaves and successful fruit set under high temperature conditions.

The genotype which shows less per cent reduction for fruit set related parameters from controlled conditions (optimum temperature and relative humidity) to higher temperature conditions was found as more temperature resilient. Keeping these parameters in mind genotypes LCA-960 and 9608U were found to the best among all the genotypes followed by hybrid UARChH-42. These genotypes LCA960 and $9608 \mathrm{U}$ may be used by the breeders in future breeding programs to increase fruit set percentage which in turn brings higher yields even under high summer temperature. The hybrid UARChH-42 may be directly utilized for the cultivation under high temperature stress condition.

\section{References}

Alberto, G. Z., Erick, S. C., Luna-Ortega, J. G., Rebeca, P. M., Juan, C., Rodríguez, O. and José L., 2013. Characterization of different capsicum varieties by evaluation of their capsaicinoids content by High Performance Liquid Chromatography, determination of pungency and effect of high temperature. Molecules, 18: 1347113486.

Bates, L. S., Waldeen, R. P. and Teare, I. D., 1973, Rapid determination of free proline in water stresses studies. Plant Soil, 39: 205-207.
Bray, H. G. and Thorpe, W. Y., 1954, Analysis of phenolic compounds of interest of metabolism. Methods Biochem. Anal., 1: 27-52.

Burton, G. W. and De Vane, E. M., 1953. Estimating heritability in tall fescue (Festuca circunclinaceae) from replicated clonal material. Agron. J. 45: 478-481.

Dahal, K. C., Sharma, M. Dhakal, D. D. and Shakya, S. M., 2006. Evaluation of heat tolerant chilli (Capsicum annuum L.) genotypes in western terai of Nepal.

J. Inst. Agric. Anim. Sci. 27: 59-64.

Demers, D. A., Yelle, S. and Gosselin, A., 1994. Effects of continuous lighting on enzyme activities of leaf carbon metabolism of tomato and pepper plants. Hort. Sci. 30(4): 250.

Dipendra, G. and Gautam, B. P., 2002. Variability, heritability and genetic advance in chilli (Capsicum spp.) Agric. Sci. Dig. 22(2): 102-104.

Federer, W. T., 1977. Experimental Design: Theory and application. McMillan, New York.

Hanson, G. H., Robinson, H. F. and Comstack, R. E., 1953. Biometrical studies of yield in segregating populations of Korian Lespedeza. Agron. J. 48: 267-282.

Johanson, R. E., Robinson, H. W. and Comstock, H. F. 1955. Estimates of genetic and environmental variability in soybean. Agron. J. 47: 314-318.

Mohammed, I., Ganiger, V. M. and Yenjerappa, S. T. 2001. Genetic variability, heritability, genetic advance and correlation studies in chilli. Karnataka J. Agri. Sci. 14(3): 784-787.

Navita, G., Jaspreet, K., Jindal, S. K., Dhaliwal, M. S. and Kanchan, P. 2016. Physiological and biochemical response to higher temperature stress 
in

hot pepper (Capsicum annuum L.). J. App. Natural Sci. 8(3): 1133- 1137.

Palacio, J. J., 1977, Spectro-photometric determination of capsaicin. J. Assoc. Off. Anal. Chem., 60(4): 970-972.

Ranganna, S., 1977, Manual analysis of fruit and vegetable products, Tata McGraw
Hill Publishing Co. Ltd., New Delhi, India, pp. 81-85.

Saha, S. R., Hossain, M. M., Rahman, M. M., Kuo, C. G. and Abdullah, S. 2010. Effect of high temperature stress on the performance of twelve sweet pepper genotypes ISSN 0258-7122. Bangladesh J. Agril. Res. 35(3): 525534.

\section{How to cite this article:}

Dileep Kumar, G. D. Tembhurne, B. V. Vikas, V. Kulkarni, R. V. Beldhadi, G. C. Shekar and Kisan, B. 2020. Screening for Temperature Tolerant Genotypes and Genetic Parameter Studies in Chilli (Capsicum annuum L.). Int.J.Curr.Microbiol.App.Sci. 9(01): 534-541. doi: https://doi.org/10.20546/ijcmas.2020.901.059 